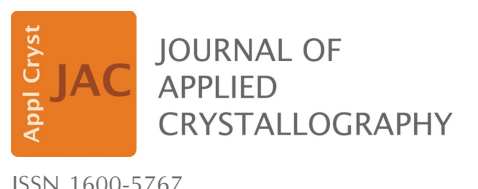

ISSN 1600-5767

Received 20 April 2015

Accepted 25 September 2015

Edited by G. Renaud, CEA-Grenoble DSM/ INAC/SP2M/NRS, Grenoble, France

Keywords: X-ray diffraction; nanowires; solar cells; ptychography.

\section{Simultaneous high-resolution scanning Bragg contrast and ptychographic imaging of a single solar cell nanowire}

\author{
Jesper Wallentin, ${ }^{*}$ Robin N. Wilke, Markus Osterhoff and Tim Salditt
}

Institute for X-Ray Physics, University of Göttingen, Friedrich-Hund-Platz 1, Göttingen, 37077, Germany. *Correspondence e-mail: jwallen@uni-goettingen.de

Simultaneous scanning Bragg contrast and small-angle ptychographic imaging of a single solar cell nanowire are demonstrated, using a nanofocused hard X-ray beam and two detectors. The $2.5 \mu \mathrm{m}$-long nanowire consists of a single-crystal InP core of $190 \mathrm{~nm}$ diameter, coated with amorphous $\mathrm{SiO}_{2}$ and polycrystalline indium tin oxide. The nanowire was selected and aligned in real space using the small-angle scattering of the $140 \times 210 \mathrm{~nm}$ X-ray beam. The orientation of the nanowire, as observed in small-angle scattering, was used to find the correct rotation for the Bragg condition. After alignment in real space and rotation, high-resolution (50 $\mathrm{nm}$ step) raster scans were performed to simultaneously measure the distribution of small-angle scattering and Bragg diffraction in the nanowire. Ptychographic reconstruction of the coherent small-angle scattering was used to achieve sub-beam spatial resolution. The small-angle scattering images, which are sensitive to the shape and the electron density of all parts of the nanowire, showed a homogeneous profile along the nanowire axis except at the thicker head region. In contrast, the scanning Bragg diffraction microscopy, which probes only the single-crystal InP core, revealed bending and crystalline inhomogeneity. Both systematic and non-systematic real-space movement of the nanowire were observed as it was rotated, which would have been difficult to reveal only from the Bragg scattering. These results demonstrate the advantages of simultaneously collecting and analyzing the small-angle scattering in Bragg diffraction experiments.

\section{Introduction}

Semiconductor nanowires are crystalline nanostructures which are intensely researched in areas such as electronics (Tomioka et al., 2012), light-emitting devices (Duan et al., 2003) and quantum physics (Mourik et al., 2012). In particular, nanowire-based solar cells have shown strong development in recent years (Wallentin et al., 2013; Colombo et al., 2009; Yao et al., 2014; Goto et al., 2009; Borgström et al., 2011). An electrical connection must be made to the as-grown nanowires to complete the device, which is normally done by depositing noncrystalline thin films of insulators and conductors. Such films can induce strong strain in the semiconductor (Bouwes Bavinck et al., 2012), which can affect for instance the electronic bandgap (Bouwes Bavinck et al., 2012) and the free carrier mobility (Lee et al., 2005).

Transmission electron microscopy can be used to investigate strain in bare nanowires (Larsson et al., 2007), but the strong absorption of electrons makes this method challenging for processed nanowire devices. X-ray diffraction (XRD) has been a key method in the study of crystalline materials for a century, and the long penetration length of X-rays allows investigations of complete devices (Hrauda et al., 2011). Owing to the difficulty in making efficient X-ray optics, XRD 
has traditionally been used to probe relatively large sample areas. With recent developments in high-brilliance synchrotron radiation sources and X-ray focusing optics, the realspace resolution has increased. It has become possible to probe many types of individual nanostructures (Hrauda et al., 2011; Mocuta et al., 2008; Williams et al., 2003; Chamard et al., 2008; Newton et al., 2009; Hanke et al., 2008; Robinson \& Harder, 2009), which can differ substantially from the sample average (Mocuta et al., 2008). Lattice changes due to strain (Mastropietro et al., 2013; Newton et al., 2009; Hrauda et al., 2011), materials composition (Haag et al., 2013; Hrauda et al., 2011), heating (Haag et al., 2013) and crystal polytypes (FavreNicolin et al., 2010; Chamard et al., 2008) have been investigated. For composite devices such as the one studied here, a limitation of Bragg scattering is that it does not give information about noncrystalline regions.

A key challenge with Bragg scattering of nanostructures is to align the sample and the beam in both real and reciprocal space (Stangl $e t$ al., 2014; Robinson \& Harder, 2009). The real-space alignment requires positioning and controllably moving the sample in the nanofocused beam, with a precision that should be significantly smaller than the focus size. The reciprocal-space requirement means that the beam and the sample lattice must be aligned at the correct angle to fulfil the Bragg condition. To collect rocking curves the sample is rotated in a small angular range around a Bragg peak. Since the sample can never be perfectly positioned at the center of rotation, this leads to a shift in real space. As the sample feature sizes and X-ray foci shrink far below $1 \mu \mathrm{m}$, the alignment problem becomes increasingly severe.

Alignment for small-angle (forward) $\mathrm{X}$-ray scattering is easier, since the positioning only has to be carried out in real space. It is based on scattering from all electrons in the sample, without interference induced by crystal planes, and essentially measures the projected electron density. Small-angle scattering can therefore give complementary information to the Bragg scattering (Gallagher-Jones et al., 2014; Bunk et al., 2009). Here, we combine smallangle and Bragg scattering to investigate a solar cell nanowire with a nanofocused X-ray beam. Ptychographic reconstruction of the small-angle scattering was used to find and track the real-space position with sub-beam-size spatial resolution. While the smallangle scattering shows a quite homo- geneous profile, the scanning Bragg diffraction microscopy reveals strong inhomogeneity in the crystalline InP core.

\section{Experimental}

The nanowire investigated here was taken from a solar cell, in which about four million nanowires in a dense matrix $(0.47 \mu \mathrm{m}$ pitch) make up a $1 \times 1 \mathrm{~mm}$ device. The nanowire solar cell preparation has been described previously (Wallentin et al., 2013; Borgström et al., 2011). Briefly, gold seed particles were first defined by nanoimprint lithography on an InP substrate. Then, $190 \mathrm{~nm}$-diameter InP nanowires doped with an axial p-i$\mathrm{n}$ junction were grown to a length of about $2.5 \mu \mathrm{m}$, after which the gold seed particles were removed by wet etching. To create a top contact, an insulating $\mathrm{SiO}_{2}$ film was deposited by atomic layer deposition to prevent axial short-circuiting, and a sunlight-transparent top contact of indium tin oxide (ITO) was
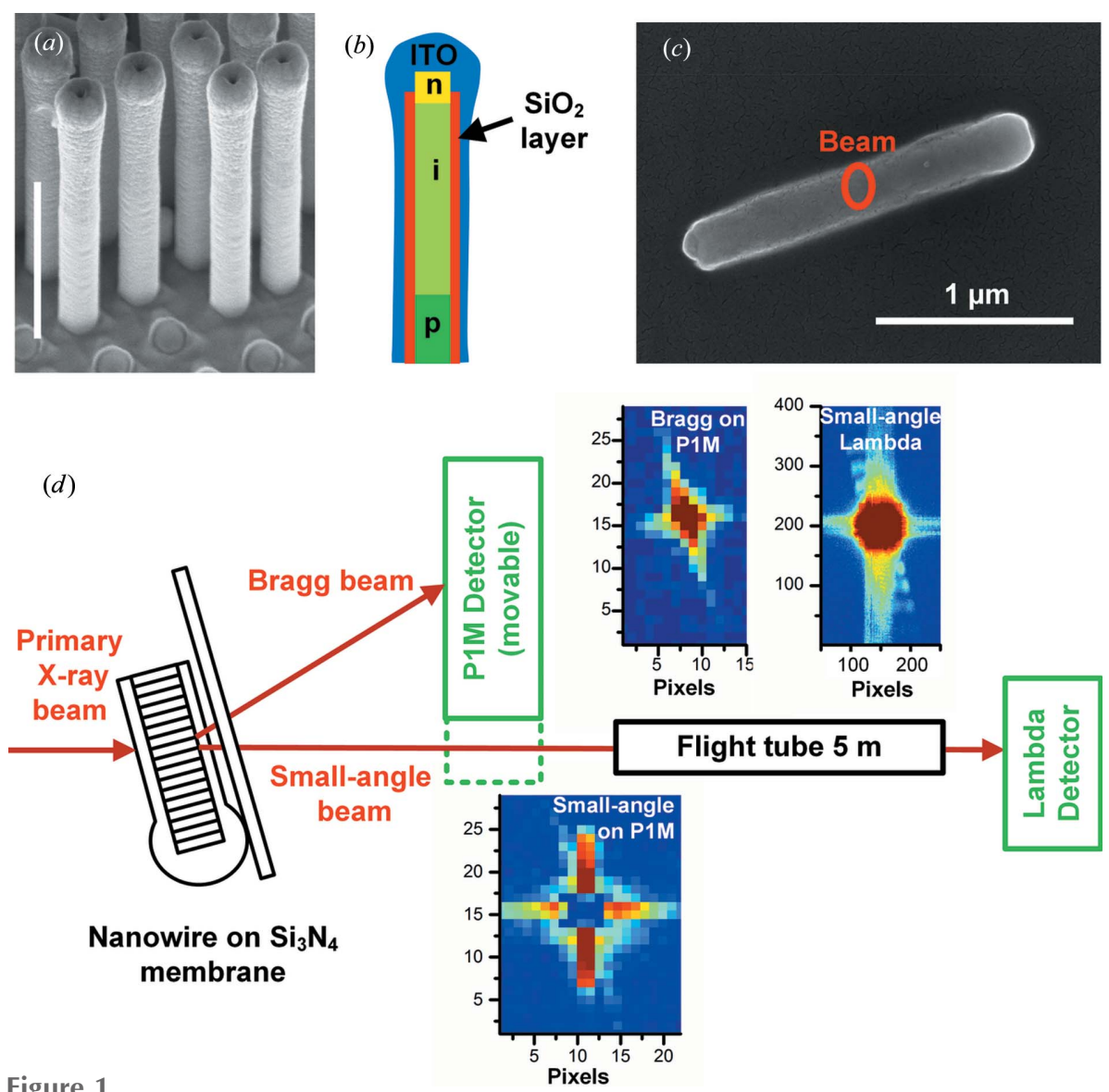

(a) Scanning electron microscopy image of nanowires in a solar cell, standing on the growth substrate. Scale bar $1 \mu \mathrm{m}$, tilt $45^{\circ}$. Stubs from broken off nanowires can be seen in the lower part. $(b)$ Drawing depicting the crystalline nanowire with its p-i-n doping structure, the amorphous $\mathrm{SiO}_{2}$ film and the polycrystalline ITO layer. The thicknesses of the layers have been exaggerated for clarity. $(c)$ Scanning electron microscopy image of the nanowire that was investigated for this paper, lying on the $\mathrm{Si}_{3} \mathrm{~N}_{4}$ membrane, taken after the experiments. The red ring shows the size of the nanofocused X-ray beam. $(d)$ Drawing showing the experimental setup, as seen from above. Two detectors were used, of which the Pilatus $1 \mathrm{M}$ closer to the sample could be moved in the horizontal direction, in and out of the small-angle beam. Examples of parts of detector images are shown, with the scale in pixels. The color scales have been adjusted to enhance the visibility of the streaks from the nanowire, which makes the high-intensity central regions appear oversaturated. In the small-angle detector images there are vertical and horizontal streaks from the KB mirrors. 
sputter deposited. The ITO sputtering gives an inhomogeneous coverage of the nanowire, with a thicker layer at the top (Figs. $1 a$ and $1 b$ ). Thus, the nanowires consist of a singlecrystal InP core, an amorphous $\mathrm{SiO}_{2}$ film and a polycrystalline ITO layer. Similar devices have shown an efficiency of $13.8 \%$ (Wallentin et al., 2013), while this cell showed an efficiency of $9 \%$.

For the X-ray investigations, nanowires were scraped off the growth substrate with clean-room tissue and deposited randomly on an X-ray-transparent $1 \mu \mathrm{m}$-thick $\mathrm{Si}_{3} \mathrm{~N}_{4}$ membrane (Fig. 1c). The GINIX (Göttingen Instrument for Nano-Imaging with X-rays) end station at the P10 beamline at the PETRA III synchrotron, DESY, Hamburg, Germany, was used for the X-ray investigations (Fig. 1d) (Salditt et al., 2015). The beam of energy $13.8 \mathrm{keV}$ was focused using KirkpatrickBaez (KB) mirrors to a size of (FWHM of the intensity) $140 \times$ $210 \mathrm{~nm}$ in the horizontal and vertical directions, respectively, as determined by ptychographic reconstruction [see below and Wilke et al. (2014)]. The real-space coordinate system $(x, y, z)$ is defined in Fig. 2. A sample stage optimized for tomography was used, rather than a diffractometer, with a single rotation around the vertical $z$ axis $\left(\theta_{z}\right)$ and high-precision piezo motors for translation.

Two different single-photon counting detectors were used, as shown in Fig. $1(d)$. Near the sample, at $x=0.38 \mathrm{~m}$, there was a Dectris Pilatus $1 \mathrm{M}$ detector $(\mathrm{P} 1 \mathrm{M})$, with $172 \mu \mathrm{m}$ pixel size, which could be moved in and out of the small-angle beam. There was also a new Lambda detector based on the Medipix 3

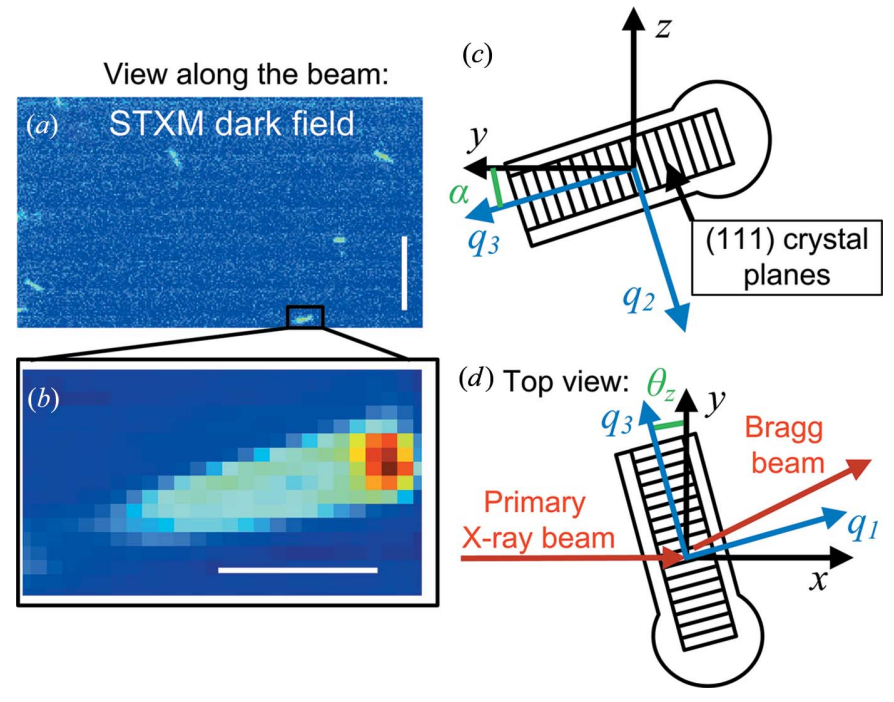

Figure 2

Rotational alignment. (a) Overview dark-field STXM image of the nanowire sample. Scale bar $10 \mu \mathrm{m}$, step size $200 \mathrm{~nm}$. Several nanowires can be seen lying on the $\mathrm{Si}_{3} \mathrm{~N}_{4}$ membrane. The horizontal stripes are due to variations in the synchrotron ring current. (b) High-resolution darkfield STXM image of a single nanowire from the boxed area in $(a)$. Scale bar $1 \mu \mathrm{m}$, step size $100 \mathrm{~nm}$. (c) Drawing depicting the nanowire as seen facing the beam. The (111) planes are oriented orthogonal to the long axis. Therefore, the scattering vector $\mathbf{q}$ is parallel to the nanowire long axis. The angle between the nanowire and the horizontal $y$ axis is $\alpha=16^{\circ}$. (d) Drawing depicting the nanowire as seen from above. The sample was rotated around the vertical $z$ axis. The rotation around the vertical $z$ axis, $\theta_{z}$, needed to meet the Bragg condition is larger than the Bragg angle, $\theta_{\mathbf{B}}$, owing to the rotation $\alpha$. chip with $55 \mu \mathrm{m}$ pixel size (Pennicard et al., 2013; Wilke et al., 2014) positioned at $x=5.1 \mathrm{~m}$, behind a flight tube, which was used together with a semi-transparent central stop to improve the dynamic range (Wilke et al., 2013).

\section{Alignment}

Different strategies have been used to align nanocrystals for Bragg scattering experiments. X-ray fluorescence maps can be used for real-space alignment if the sample has a suitable absorption edge (Hanke et al., 2008). If epitaxically grown nanocrystals are investigated on the growth substrate, the sample can first be aligned in reciprocal space, by using the strong substrate Bragg reflection (Mocuta et al., 2008). After alignment in reciprocal space, the sample can then be scanned in real space until a single nanostructure is found. In many cases, such as the present one, the substrate and the nanocrystal are of the same material, which means that the nanocrystal signal would have to be detected against a much stronger substrate signal. Furthermore, in order to measure single nanowires in such a dense nanowire array, a diffraction angle close to the nanowire axis would have had to be used. The diffraction signal would then have been averaged over a large segment of the nanowire, degrading the spatial resolution with regards to the optimum size given by the X-ray beam focus.

Instead, we chose to measure single nanowires, which had been broken off from the growth substrate, and to start with the real-space alignment. This approach completely removes any scattering from the growth substrate and allowed using a low-index Bragg reflection. This method should preserve the nanowires in their original strain state, except very near the base, since the processing layers remain intact, but further experiments comparing standing and scraped off nanowires should be performed to verify this. After coarse alignment with an in-line optical microscope, we made a large-area scanning transmission X-ray microscopy (STXM) image with sufficient resolution to reveal individual nanowires (step size $200 \mathrm{~nm}$; Fig. 2a). Then, a single nanowire was chosen and imaged with a smaller STXM image, step size $100 \mathrm{~nm}$, as shown in Fig. 2(b). In STXM, the sample is two-dimensional raster scanned in the beam, and detector images are acquired at each point. From these images, different contrast modes can be evaluated, as discussed in $\S 4$.

Since the rotational alignment could not be carried out using a substrate peak, the measured shape of the nanowires was used instead. InP nanowires grow in the cubic zincblende (111)B direction, with the (111) crystal planes oriented orthogonal to the long axis (Fig. 2c) (Hiruma et al., 1995). During crystal growth, a high density of wurtzite crystal segments are also formed, so the nanowire is a polytypic mixture. We were nominally measuring the Bragg reflection for the zincblende (111) planes, whose scattering vector is parallel with and only $0.36 \%$ larger than that of the wurtzite 000.2 reflection (Kriegner et al., 2011). For simplicity, we refer only to the cubic 111 reflection. The 111 reflection has a Bragg angle of $\theta_{\mathrm{B}}=7.62^{\circ}$ at X-ray energy $13.8 \mathrm{keV}$. The small angle, 
almost orthogonal to the nanowire axis, preserved the high spatial resolution of the nanofocused beam. The rotational alignment of the membrane was first done with the membrane parallel with the beam $\left(\theta_{z}=90^{\circ}\right)$, using knife-edge scans.

The correct $\theta_{z}$ for the Bragg condition is not $\theta_{\mathrm{B}}$, since the nanowire was rotated in the plane of the sample membrane. However, the in-plane rotation $\alpha$ for the chosen nanowire could be readily evaluated from the STXM scans. Since the (111) planes are orthogonal to, and the scattering vector $\mathbf{q}$ is parallel with, the nanowire long axis, the rotation is (Appendix A) $\theta_{z}=\arcsin (\lambda / 2 d \cos \alpha)$. With the measured $\alpha=16^{\circ}$, we calculated $\theta_{z}=8.0^{\circ}$. Rocking curve measurements were performed (see Fig. $6 a$ in $\$ 5$ ), giving a peak value of $10.88^{\circ}$. The deviation is presumably because the ITO film on the nanowire was thicker at the top, which meant that the nanowire axis was not perfectly aligned with the membrane. The geometrical calculation also predicts the vertical and horizontal deflection of the Bragg peak on the detector, which was $(y, z)=(9.88,3.07) \mathrm{cm}$ compared with the measured value $(9.89,2.81) \mathrm{cm}$. Since the Bragg angle can be accurately predicted it would be possible to use a small high-resolution detector placed further away for increased angular resolution.

In Fig. 1 $(d)$, examples of the regions of interest (ROI) in detector images are shown. In both small-angle and Bragg scattering, the diffraction peaks are to first order a convolution of the Fourier transforms of the probe beam and the shape function of the object (Hruszkewycz et al., 2012; Robinson et al., 2001). The shape function is defined as 1 in the object and 0 outside of the object. For a rod-shaped object, the twodimensional Fourier transform of the shape function generates streaks orthogonal to the long axis of the rod. Streaks orthogonal to the nanowire axis can therefore be seen in all three detector images. In the small-angle scattering image, vertical and horizontal streaks from the KB mirrors can also be observed.

\section{Multi-mode imaging}

After alignment of the sample in both real and reciprocal space, STXM scans were performed with the P1M detecting the Bragg peak and the Lambda detector measuring the smallangle scattering (step length $100 \mathrm{~nm}$, exposure time $1 \mathrm{~s}$ per point). Several imaging contrast modes can be calculated from the small-angle scattering (Fig. 3) (Thibault et al., 2009; Bunk et al., 2009), all of which probe both crystalline and noncrystalline parts of the sample. The transmission (bright-field) STXM is here defined as the total intensity in the small-angle scattering ROI, corresponding to the size of the propagated beam on the detector. This signal is modulated by absorption in the object. Conversely, dark-field STXM is here defined as the total signal outside of the same ROI. It is therefore a measure of the amount of scattered light. Differential phase contrast (DPC) is based on an evaluation of the center of mass (COM) of the scattered signal on the detector, which is modified by density gradients in the object.

While the contrast in transmission STXM is poor (Fig. 3a), about $0.5 \%$, the dark-field image shows about $20 \%$ contrast
(Fig. 3b). The DPC images show contrast at the nanowire edges (Figs. $3 c$ and $3 d$ ), where there is a gradient in electron density. Since the nanowire was oriented almost horizontally, the shape of the nanowire is most easily seen in the vertical DPC.

The Bragg signal on the P1M detector was analyzed per spatial point to create a two-dimensional image (Mocuta et al., 2008). We use the term scanning Bragg diffraction microscopy (SBM) for this method, to distinguish it from the scanning small-angle diffraction methods which have different contrast mechanisms (Thibault et al., 2008). The SBM image in Fig. 3(e) was achieved by integrating the intensity of the Bragg peak ROI, per spatial point, similar to transmission STXM. The SBM image shows the spatial distribution of the Bragg scattering for a particular angle and only probes the single-crystal InP core. It shows major differences compared with the images from small-angle scattering, with two strongly scattering regions in the middle and lower end, but no signal from the top part of the nanowire. The spatial resolution in all these imaging modes is limited by the size of the focused beam, but coherent imaging techniques can reach sub-beam-size resolution by iteratively reconstructing the phase (Miao et al., 1999).

A requirement for such reconstruction methods, however, is that the scattered beam is sufficiently oversampled (Miao et

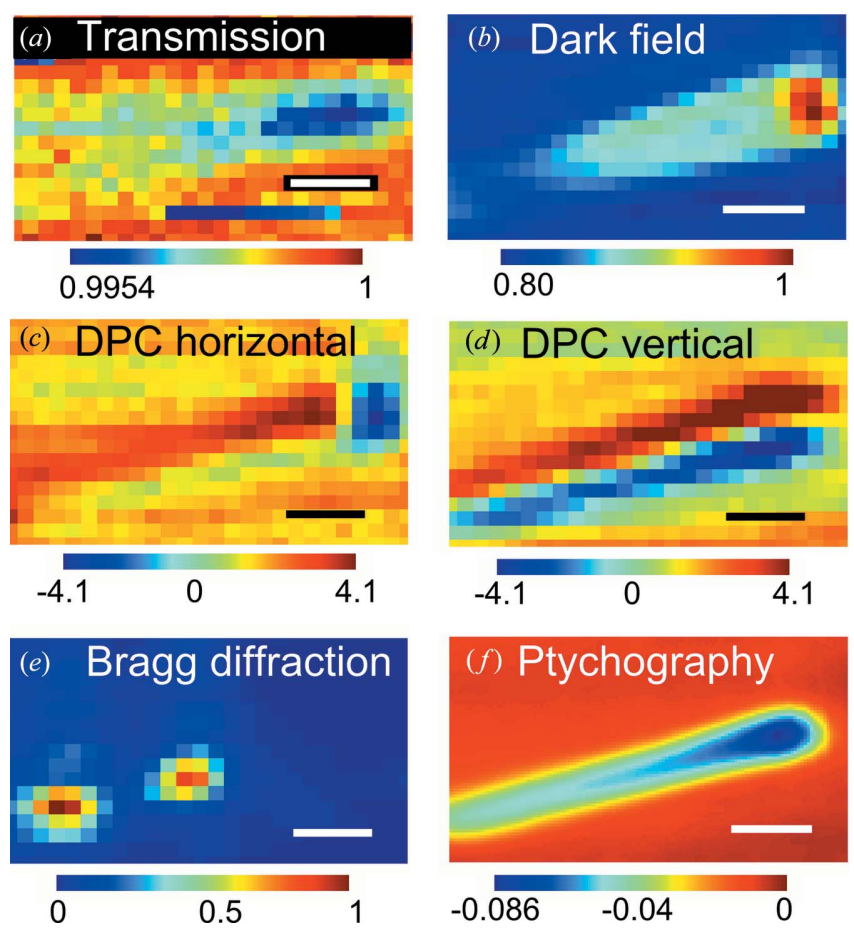

Figure 3

Multimode imaging analysis of a single scan. Step size $100 \mathrm{~nm}$, scale bars $0.5 \mu \mathrm{m}$. (a) Transmission STXM, normalized intensity. The data were modified to compensate for systematic intensity fluctuations due to synchrotron ring top-ups. (b) Dark-field STXM image, normalized intensity. (c) Differential phase contrast in the horizontal direction, in $10^{6} \mathrm{rad} \mathrm{m}^{-1}$. (d) Differential phase contrast in the vertical direction, in $10^{6} \mathrm{rad} \mathrm{m}^{-1}$. (e) Scanning Bragg diffraction microscopy, normalized intensity. $(f)$ Ptychographic reconstruction, phase shift in rad. 
al., 1998). In single-exposure coherent diffraction imaging, the oversampling ratio, $\sigma$, should be larger than two. For a onedimensional object of size $L=200 \mathrm{~nm}$, X-ray wavelength $\lambda=$ $0.899 \AA$, detector distance $z_{\mathrm{D}}$ and pixel size $x_{\mathrm{D}}$, we have in the far-field geometry $\sigma=z_{\mathrm{D}} \lambda / L x_{\mathrm{D}}$. For the small-angle scattering at the Lambda detector, we get $\sigma \simeq 42$, while for the Bragg scattering at the $\mathrm{P} 1 \mathrm{M}$ we find $\sigma \simeq 1$. We employed an alternative coherent imaging method, ptychography, where the object is scanned in the beam with an overlapping exposure between different positions (Rodenburg et al., 2007). It is more complicated to define the sampling ratio for ptychography owing to the overlapping exposures, and the criterion could be considerably relaxed with this method (Edo et al., 2013). The one-dimensional sampling ratio is still proportional to $z_{\mathrm{D}} \lambda / x_{\mathrm{D}}$, as in single-exposure CDI, which in our experiment means that it is very difficult to reconstruct the phase for the Bragg scattering. The available detectors and the geometry of the beamline did not allow better sampling of the Bragg
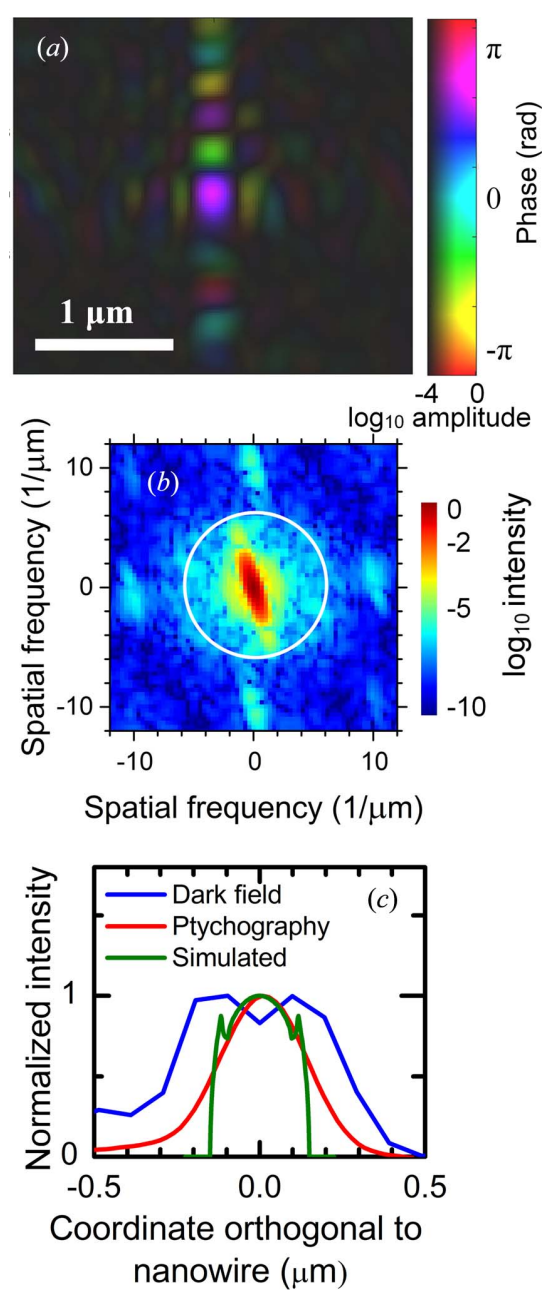

Figure 4

Ptychography. (a) Phase and amplitude of reconstructed probe beam. (b) Power spectral density of the reconstructed nanowire in Fig. 3(f). The white ring indicates $6 \mu \mathrm{m}^{-1}$. (c) Cross-sectional profiles, averaged along a section of the nanowire, and the simulated phase shift. All profiles were normalized to their maximum. scattering at the time of the experiment, but this is not a fundamental limitation of our method.

The STXM data were used to make a ptychographic reconstruction of the small-angle scattering data with the ePIE algorithm (reconstruction parameters $\alpha=\beta=0.5$ ) (Maiden \& Rodenburg, 2009; Wilke et al., 2014), which simultaneously reconstructs the object and the probe beam (Figs. $3 f$ and $4 a$ ). The resulting image has higher resolution than the STXM modes, and the thicker head can be clearly seen. In the power spectral density map of the ptychographic image (Fig. 4b) the contributions above the background extend to approximately $\nu=6 \mu \mathrm{m}^{-1}$, as indicated by a white ring, corresponding to a half-period real-space pixel size $(1 / 2 v)$ of about $80 \mathrm{~nm}$ (Shapiro et al., 2005; Wilke et al., 2014).

To get a better comparison of the spatial resolutions of the different imaging modes, averaged line profiles were calculated across the nanowires (Fig. 4c). The expected phase shift profile was also calculated, based on the nominal layer thicknesses and material data. The calculated profile is almost box shaped, with two $10 \mathrm{~nm}$-thick peaks at the edges, since the InP core and the ITO film have the highest electron density and phase shift $\left(\delta=4.6 \times 10^{-6}\right.$ and $6.9 \times 10^{-6}$, respectively $)$ and the $\mathrm{SiO}_{2}$ film has the lowest $\left(\delta=2.9 \times 10^{-6}\right)$. The FWHM of the dark-field STXM was $0.54 \mu \mathrm{m}$, which is reasonable considering a convolution of a $0.31 \mu \mathrm{m}$ object with a $0.21 \mu \mathrm{m}$ beam. The profile of the ptychographic image showed a Gaussian profile with an FWHM of $0.30 \mu \mathrm{m}$. Although this is similar to the nanowire diameter, the profile should ideally not be Gaussian shaped. Thus, the spatial resolution was not sufficient to distinguish the different layers in the core-shell structure, but was good enough to resolve variations along the nanowire axis. The SBM scans showed lower-diameter cross sections than the STXM images, which is expected since they only probe the $0.19 \mu \mathrm{m}$-diameter crystalline core. While the SBM scans in Fig. 3 had too few data points to allow a meaningful analysis, a similar analysis of the higher-resolution SBM scans (see below) showed an FWHM of $0.44 \mu \mathrm{m}$. Ptychography is quantitative, in the sense that the reconstruction gives absolute values for the phase shift which can be used to calculate the projected electron density (Giewekemeyer et al., 2010). The measured phase shift in the center of the body of the nanowire was $-44 \mathrm{mrad}$, which is only half of the calculated $-90 \mathrm{mrad}$. This discrepancy may be related to the limited resolution.

\section{Spatial variations of the scattering vector}

Next, the multimode imaging was repeated at five different rotations. The object was not perfectly positioned at the center of rotation, so that the sample therefore systematically moved in the $\mathbf{y}$ direction during rotation. Using STXM scans we could characterize and compensate for this movement, which we measured to be $7.5 \mu \mathrm{m}$ per degree. However, the ptychographic images revealed that there was residual nonsystematic real-space movement of the sample of the order of $100 \mathrm{~nm}$ per $0.05^{\circ}$ step (Fig. 5), both horizontally and vertically. Note that 
such movement would be difficult to observe only from the Bragg scattering.

In Fig. 6(a), a rocking curve around the vertical $z$ axis is shown for the point with highest Bragg intensity near the base of the nanowire, with a peak value of $\theta_{z}=10.88^{\circ}$ and an FWHM of $0.14^{\circ}$. The lower half of the nanowire was investigated with high-resolution scans (50 nm step size) at five rotations (Fig. 6): 10.78, 10.83, 10.88, 10.93 and 10.98 ${ }^{\circ}$. For each real-space point, the data were first shifted to compensate for real-space movement. The scattering intensities from all rotations were then mapped in reciprocal space. An image of the total intensity of all angles is shown in Fig. $6(b)$. The real-space variation of the position of the Bragg peak in reciprocal space, $\mathbf{q}_{\mathrm{B}}(y, z)$, can reveal minute shifts due to strain and bending (Etzelstorfer et al., 2014). We chose the measured $\mathbf{q}_{\mathrm{B}}$ in the middle of the nanowire as reference for a fixed orthogonal reciprocal-space coordinate system. The coordinate system, shown in Fig. 2, has $q_{3}$ nominally parallel with the nanowire axis, $q_{2}$ parallel with the membrane and orthogonal to the nanowire, and $q_{1}$ orthogonal to both nanowire and membrane. Thus, $q_{3}$ is the main coordinate, $\left|\mathbf{q}_{\mathrm{B}}\right| \simeq q_{3}$, while the orthogonal directions $q_{1}$ and $q_{2}$ relate to tilting of the crystal lattice. Note that $q_{1}$ and $q_{2}$ cannot be directly related to any crystal direction. At the reference point in the middle of the nanowire, $q_{1}=q_{2}=0$ and $\left|\mathbf{q}_{\mathrm{B}}\right|=q_{3}$. Gaussian peak fits were carried out to quantify the coordinates of $\mathbf{q}_{\mathrm{B}}$. The COM was also evaluated, but we found peak fitting to be more resilient to background noise. Outside of the high-intensity region, the peak fits of the $q_{1}$ component were not sufficiently reliable to analyze further. This mapping and
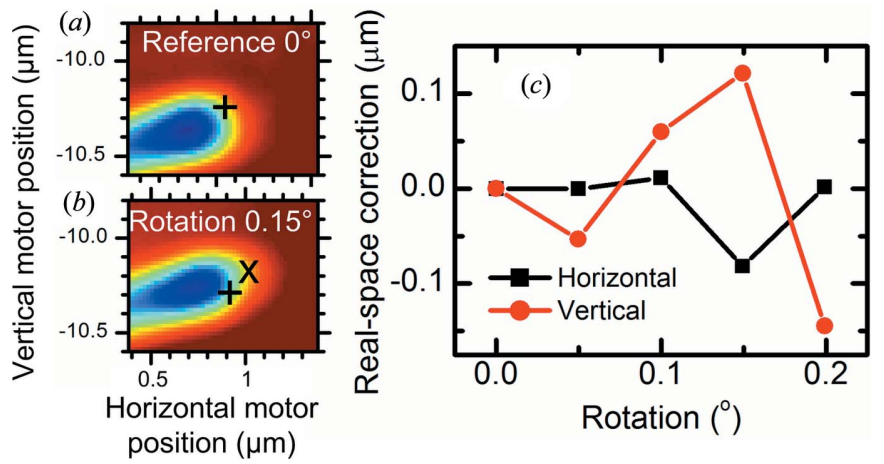

Figure 5

Measuring real-space movement due to rotation. (a) Parts of ptychographic reconstructions, such as in Fig. $3(f)$. The + sign indicates the reference point. In the direction across the nanowire, this point was calculated from the average of the intensity summed along the nanowire axis. In the direction along the nanowire axis, this point was the halfmaximum of the slope at the tip. The horizontal coordinate is the motor position. The scan ranges were adapted to the systematic movement of $7.5 \mu \mathrm{m}$ per degree. (b) Part of a ptychographic reconstruction, taken at a relative rotation around the $z$ axis of $+0.15^{\circ}$, compared with $(a)$. Here, the $\times$ sign indicates the reference point calculated from this image. The + sign indicates the expected position of this reference point, calculated from the systematic shift in the $\mathbf{y}$ direction. (c) Remaining shift of the reference point versus relative rotation, after correction for systematic movement. This is the distance between the measured (' $x$ ') and expected ('+') positions of the reference points, as in (b). peak fitting was repeated for each real-space point, giving twodimensional images of the $q$ positions (Figs. $6 c$ and $6 d$ ).

The length of $\mathbf{q}_{\mathrm{B}},\left|q_{\mathrm{B}}\right|$, which is related to the (111) lattice plane distance, was $1.832 \AA^{-1}$, which is slightly less than the calculated $1.85 \AA^{-1}$. The deviation of about $1 \%$ is probably because the detector distance was not thoroughly calibrated, since we are concerned with relative variations within the nanowire. The relative variation in $\left|\mathbf{q}_{\mathrm{B}}\right|$ along the nanowire axis was only of the order of $10^{-4}$. The $q_{2}$ component, however, showed a clear gradient along the nanowire axis, which suggests that the lattice was rotated around $q_{1}$. From the shift in $q_{2}$, the rotation around $q_{1}$ can be calculated for every spatial point as $\beta=\arctan \left(-q_{2} / q_{3}\right) \simeq-q_{2} / q_{3}$. The rotation was averaged perpendicularly to the nanowire axis and plotted versus the real-space coordinate along the nanowire, $s$ (Fig. 5c). For most of the range, there is a linear dependence which can be fitted with a gradient $\mathrm{d} \beta / \mathrm{d} s=-1.78 \mathrm{mrad} \mu \mathrm{m}^{-1}$. Therefore, the orientation in this region can be described by a single radius of curvature of $R=|\mathrm{d} s / \mathrm{d} \beta|=0.56 \mathrm{~mm}$. The real-space deflection from an ideally straight nanowire in this $1 \mu \mathrm{m}$ segment is only about $2 \mathrm{~nm}$, which is too small to observe directly.
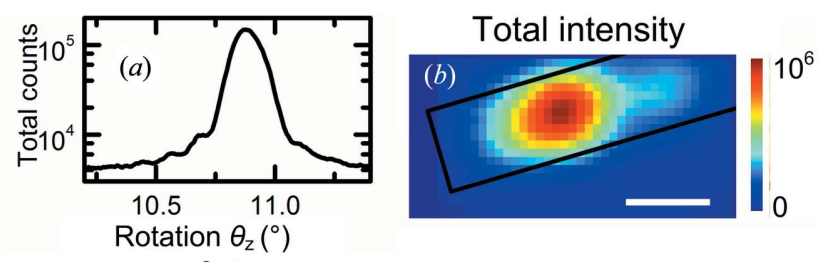

$q_{2}\left(10^{-3} / \AA\right)$
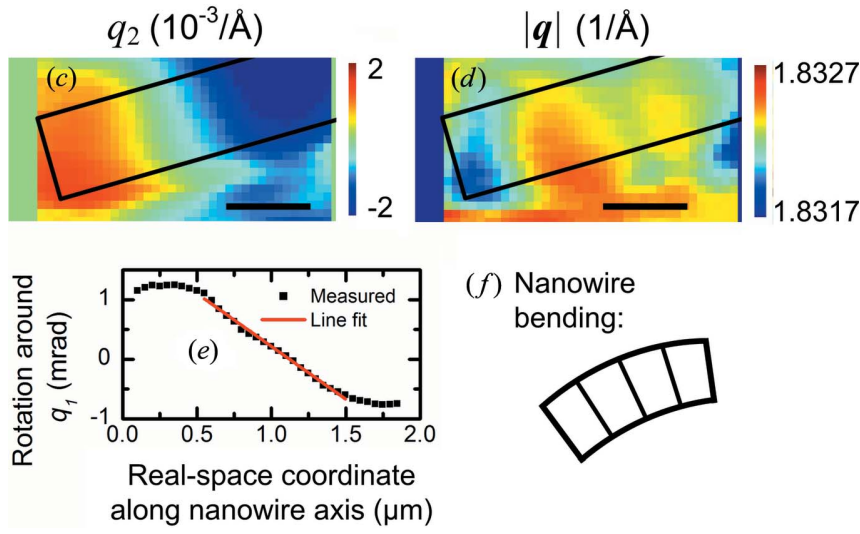

(f) Nanowire bending:

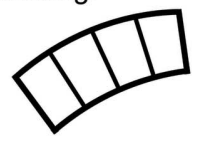

Figure 6

High-resolution scanning Bragg diffraction microscopy of the left (lower) half of the nanowire. (a) Rocking curve of $\theta_{z}$, around the $z$ axis, from the highest-intensity region near the base of the nanowire. The FWHM is $0.14^{\circ}$. (b) Sum of the intensity in the Bragg peak ROI of all angles. $(c),(d)$ Images of $q_{2}$ and $|\mathbf{q}|$, respectively. Each SBM scan was first shifted in order to compensate for real-space movement. The boxes in $(b)-(d)$ indicate the position of the nanowire, as evaluated from the small-angle scattering. Scale bars are $0.5 \mu \mathrm{m}$, step size $50 \mathrm{~nm}$. The position of the Bragg peak can be measured and analyzed also outside the boxed region, since the focused X-ray beam has intensity outside of the focus (see Fig. 4a). (e) The rotation around $q_{1}$, calculated as $\beta=-q_{2} / q_{3}$ and averaged across the nanowire, versus a real-space coordinate, $s$, along the nanowire axis (parallel with $q_{3}$ ). The red line shows a linear fit with slope $\mathrm{d} \beta / \mathrm{d} s=$ $-1.78 \mathrm{mrad} \mu \mathrm{m}^{-1}$. ( $f$ ) Exaggerated sketch of how the nanowire bends in the plane of the membrane, as seen along the beam (compare with Fig. 2c). 


\section{Discussion}

The small-angle scattering images showed a relatively homogeneous nanowire, with a thicker head region. To be able to distinguish between the different layers in the core-shell structure the real-space resolution should be improved to about $10 \mathrm{~nm}$. The SBM images showed two strongly scattering regions, positioned roughly at the base and the middle of the nanowire. The Bragg signal at the top part of the nanowire was very weak in the angular range investigated here. The reason for this inhomogeneity is unclear, but two reasons can be mentioned.

First, InP nanowires typically grow in a mix of cubic zincblende and hexagonal wurtzite crystal structure, which differ in the lattice plane spacing and therefore Bragg angle (Kriegner et al., 2011). We were nominally measuring the Bragg reflection for the zincblende (111) planes, whose scattering vector is only $0.36 \%$ larger than that of the wurtzite 000.2 reflection (Kriegner et al., 2011). The crystal structure depends on many parameters, of which doping is the most relevant here (Wallentin \& Borgström, 2011). The p-i-n doping profile leads to regions with different proportions of wurtzite and zincblende, with more zincblende at the base and less at the top, which in turn leads to variations in the lattice parameter.

Second, the ITO and $\mathrm{SiO}_{2}$ films stress the nanowire core, and since the tip has a much thicker ITO layer, the strain could be so strong that the Bragg scattering at the tip was out of the investigated angular range. The SBM method is time consuming, which unfortunately prevented us from making scans at a large range of angles. Weak Bragg scattering may also be due to broadening caused by gradients in the lattice parameter, axially or radially, since the signal is a coherent superposition of scattering from many lattice planes. The top part of the processed nanowire is characterized by steep changes in layer thicknesses, and possibly strong strain gradients.

The SBM measurements also revealed that the nanowire was bent in the plane of the membrane. We tentatively attribute this to an asymmetric thickness of the ITO film. In the scanning electron microscopy image in Fig. 1(c) and in the ptychographic image in Fig. 3(f), the head of the nanowire is slightly thicker on the lower side. This asymmetry is a geometric effect of the ITO sputtering. The side of the nanowire facing the center of the sample, and therefore the ITO source, was coated with a little more ITO, while the other side was slightly shadowed. The uneven thickness could give an asymmetric stress, which in the flexible nanowires can lead to bending. Note that the asymmetric ITO thickness, or even the orientation of the head region, could not have been identified only from the SBM images.

\section{Conclusions}

In conclusion, we have demonstrated that small-angle scattering can be used for aligning and tracking the real-space position of a nanowire with high resolution. The alignment method used here is deterministic, in the sense that a particular object can be selected and then rotated to the correct Bragg angle. Crucial for the reciprocal-space alignment in our experiment was the preexisting knowledge that the (111) planes are orthogonal to the nanowire axis. However, it is a general property of crystals to exhibit low-energy low-index facets, which means that alignment using facets could be applied to many types of nanocrystals. The measurements also demonstrate how small-angle scattering and Bragg diffraction can give complementary information about a sample, especially when it contains both crystalline and noncrystalline regions.

There are further opportunities with simultaneously collecting the Bragg and small-angle scattering which have not been explored here. In crystalline heterostructures it can be difficult to separate the effects of composition and strain without sophisticated models, since they both affect the lattice constant (Mocuta et al., 2008; Keplinger et al., 2009). Since different materials in a heterostructure have different electron density, forward scattering could be used to independently measure the composition. Another possibility is to use the reconstructed probe from small-angle scattering to improve the Bragg imaging. In recent years superresolution methods have been developed for Bragg scattering, based on solving the phase problem by iterative methods similarly to smallangle scattering (Robinson et al., 2001; Godard et al., 2011; Hruszkewycz et al., 2012; Minkevich et al., 2014). In these coherent Bragg methods, the Bragg peak is a convolution of the sample properties, like lattice tilt, lattice strain and sample shape, with the properties of the probe (Hruszkewycz et al., 2012; Godard et al., 2011). Characterizing the probe from the small-angle scattering data could decouple the probe and sample effects, and improve the reconstructions of the Bragg scattering data.

\section{APPENDIX $A$}

\section{Modified Bragg condition}

The nanowires grow in the (111)B direction, and we use the Bragg reflection from the (111) planes which are orthogonal to the growth axis. The direction of the nanowire, in the realspace coordinate system

$$
\left(\begin{array}{l}
x \\
y \\
z
\end{array}\right)
$$

is

$$
\mathbf{r}=\left(\begin{array}{c}
0 \\
\cos \alpha \\
-\sin \alpha
\end{array}\right)
$$

To fulfill the Bragg condition, we rotate the sample around the $z$ axis by the angle $\theta_{z}$. The direction of the rotated nanowire is then 
$\mathbf{r}=\left(\begin{array}{ccc}\cos \theta_{z} & -\sin \theta_{z} & 0 \\ \sin \theta_{z} & \cos \theta_{z} & 0 \\ 0 & 0 & 1\end{array}\right)\left(\begin{array}{c}0 \\ \cos \alpha \\ -\sin \alpha\end{array}\right)=\left(\begin{array}{c}-\sin \theta_{z} \cos \alpha \\ \cos \theta_{z} \cos \alpha \\ -\sin \alpha\end{array}\right)$

For the (111) planes, the reciprocal lattice vector $\mathbf{G}$ with length $2 \pi / d$ is parallel to the nanowire axis. Thus,

$$
\mathbf{G}=\frac{2 \pi}{d}\left(\begin{array}{c}
-\sin \theta_{z} \cos \alpha \\
\cos \theta_{z} \cos \alpha \\
-\sin \alpha
\end{array}\right)
$$

The incident wavevector of the primary beam is

$$
\mathbf{k}_{\mathrm{i}}=\frac{2 \pi}{\lambda}\left(\begin{array}{l}
1 \\
0 \\
0
\end{array}\right) \text {. }
$$

The Bragg condition can be written as

$$
\begin{gathered}
2 \mathbf{k}_{\mathrm{i}} \cdot \mathbf{G}=\mathbf{G}^{2}, \quad \frac{-8 \pi^{2} \sin \theta_{z} \cos \alpha}{d \lambda}=\frac{4 \pi^{2}}{d^{2}}, \\
\theta_{z}= \pm \arcsin \left(\frac{\lambda}{2 d \cos \alpha}\right) .
\end{gathered}
$$

Since the scattered wavevector $\mathbf{k}_{\mathrm{f}}=\mathbf{k}_{\mathrm{i}}+\mathbf{G}$, this can also be used to calculate the direction of the Bragg reflection and the expected position at the detector.

\section{Acknowledgements}

We thank Mike Kanbach for the preparation of the semitransparent central stop and Magnus Borgström, Damir Asoli, Maria Huffman, Ingvar Åberg and Martin Magnusson for the nanowire solar cell sample. Financial support by the K. A. Wallenberg Foundation and SFB 755 'Nanoscale Photonic Imaging' of the Deutsche Forschungsgemeinschaft is gratefully acknowledged.

\section{References}

Borgström, M. T., Wallentin, J., Heurlin, M., Fält, S., Wickert, P., Leene, J., Magnusson, M. H., Deppert, K. \& Samuelson, L. (2011). Quantum Electron. 17, 1050-1061.

Bouwes Bavinck, M., Zieliński, M., Witek, B. J., Zehender, T., Bakkers, E. P. A. M. \& Zwiller, V. (2012). Nano Lett. 12, 6206-6211.

Bunk, O., Bech, M., Jensen, T. H., Feidenhans'l, R., Binderup, T., Menzel, A. \& Pfeiffer, F. (2009). New J. Phys. 11, 123016.

Chamard, V., Stangl, J., Labat, S., Mandl, B., Lechner, R. T. \& Metzger, T. H. (2008). J. Appl. Cryst. 41, 272-280.

Colombo, C., Heiß, M., Grätzel, M. \& Fontcuberta i Morral, A. (2009). Appl. Phys. Lett. 94, 173108.

Duan, X. F., Huang, Y., Agarwal, R. \& Lieber, C. M. (2003). Nature, 421, 241-245.

Edo, T. B., Batey, D. J., Maiden, A. M., Rau, C., Wagner, U., Pešić, Z. D., Waigh, T. A. \& Rodenburg, J. M. (2013). Phys. Rev. A, 87, 053850 .

Etzelstorfer, T., Süess, M. J., Schiefler, G. L., Jacques, V. L. R., Carbone, D., Chrastina, D., Isella, G., Spolenak, R., Stangl, J., Sigg, H. \& Diaz, A. (2014). J. Synchrotron Rad. 21, 111-118.

Favre-Nicolin, V., Mastropietro, F., Eymery, J., Camacho, D., Niquet, Y. M., Borg, B. M., Messing, M. E., Wernersson, L. E., Algra, R. E., Bakkers, E. P. A. M., Metzger, T. H., Harder, R. \& Robinson, I. K. (2010). New J. Phys. 12, 035013.
Gallagher-Jones, M. et al. (2014). Nat. Commun. 5, 3798.

Giewekemeyer, K., Thibault, P., Kalbfleisch, S., Beerlink, A., Kewish, C. M., Dierolf, M., Pfeiffer, F. \& Salditt, T. (2010). Proc. Natl Acad. Sci. USA, 107, 529-534.

Godard, P., Carbone, G., Allain, M., Mastropietro, F., Chen, G., Capello, L., Diaz, A., Metzger, T. H., Stangl, J. \& Chamard, V. (2011). Nat. Commun. 2, 568.

Goto, H., Nosaki, K., Tomioka, K., Hara, S., Hiruma, K., Motohisa, J. \& Fukui, T. (2009). Appl. Phys. Expr. 2, 035004.

Haag, S. T., Richard, M.-I., Welzel, U., Favre-Nicolin, V., Balmes, O., Richter, G., Mittemeijer, E. J. \& Thomas, O. (2013). Nano Lett. 13, 1883-1889.

Hanke, M., Dubslaff, M., Schmidbauer, M., Boeck, T., Schöder, S., Burghammer, M., Riekel, C., Patommel, J. \& Schroer, C. G. (2008). Appl. Phys. Lett. 92, 193109.

Hiruma, K., Yazawa, M., Katsuyama, T., Ogawa, K., Haraguchi, K., Koguchi, M. \& Kakibayashi, H. (1995). J. Appl. Phys. 77, 447-462.

Hrauda, N., Zhang, J., Wintersberger, E., Etzelstorfer, T., Mandl, B., Stangl, J., Carbone, D., Holý, V., Jovanović, V., Biasotto, C., Nanver, L. K., Moers, J., Grützmacher, D. \& Bauer, G. (2011). Nano Lett. 11, 2875-2880.

Hruszkewycz, S. O., Holt, M. V., Murray, C. E., Bruley, J., Holt, J., Tripathi, A., Shpyrko, O. G., McNulty, I., Highland, M. J. \& Fuoss, P. H. (2012). Nano Lett. 12, 5148-5154.

Keplinger, M., Mårtensson, T., Stangl, J., Wintersberger, E., Mandl, B., Kriegner, D., Holý, V., Bauer, G., Deppert, K. \& Samuelson, L. (2009). Nano Lett. 9, 1877-1882.

Kriegner, D., Wintersberger, E., Kawaguchi, K., Wallentin, J., Borgström, M. T. \& Stangl, J. (2011). Nanotechnology, 22, 425704.

Larsson, M. W., Wagner, J. B., Wallin, M., Håkansson, P., Fröberg, L. E., Samuelson, L. \& Wallenberg, L. R. (2007). Nanotechnology, 18, 015504.

Lee, M. L., Fitzgerald, E. A., Bulsara, M. T., Currie, M. T. \& Lochtefeld, A. (2005). J. Appl. Phys. 97, 011101.

Maiden, A. M. \& Rodenburg, J. M. (2009). Ultramicroscopy, 109, 1256-1262.

Mastropietro, F., Eymery, J., Carbone, G., Baudot, S., Andrieu, F. \& Favre-Nicolin, V. (2013). Phys. Rev. Lett. 111, 215502.

Miao, J., Charalambous, P., Kirz, J. \& Sayre, D. (1999). Nature, 400, 342-344.

Miao, J., Sayre, D. \& Chapman, H. N. (1998). J. Opt. Soc. Am. A, 15, 1662-1669.

Minkevich, A. A., Köhl, M., Escoubas, S., Thomas, O. \& Baumbach, T. (2014). J. Synchrotron Rad. 21, 774-783.

Mocuta, C., Stangl, J., Mundboth, K., Metzger, T. H., Bauer, G., Vartanyants, I. A., Schmidbauer, M. \& Boeck, T. (2008). Phys. Rev. $B, \mathbf{7 7}, 245425$.

Mourik, V., Zuo, K., Frolov, S. M., Plissard, S. R., Bakkers, E. P. A. M. \& Kouwenhoven, L. P. (2012). Science, 336, 1003-1007.

Newton, M. C., Leake, S. J., Harder, R. \& Robinson, I. K. (2009). Nat. Mater. 9, 120-124.

Pennicard, D., Lange, S., Smoljanin, S., Hirsemann, H., Graafsma, H., Epple, M., Zuvic, M., Lampert, M. O., Fritzsch, T. \& Rothermund, M. (2013). J. Phys. Conf. Ser. 425, 062010.

Robinson, I. \& Harder, R. (2009). Nat. Mater. 8, 291-298.

Robinson, I. K., Vartanyants, I. A., Williams, G. J., Pfeifer, M. A. \& Pitney, J. A. (2001). Phys. Rev. Lett. 87, 195505.

Rodenburg, J. M., Hurst, A. C., Cullis, A. G., Dobson, B. R., Pfeiffer, F., Bunk, O., David, C., Jefimovs, K. \& Johnson, I. (2007). Phys. Rev. Lett. 98, 034801.

Salditt, T., Osterhoff, M., Krenkel, M., Wilke, R. N., Priebe, M., Bartels, M., Kalbfleisch, S. \& Sprung, M. (2015). J. Synchrotron Rad. 22, 867-878.

Shapiro, D., Thibault, P., Beetz, T., Elser, V., Howells, M., Jacobsen, C., Kirz, J., Lima, E., Miao, H., Neiman, A. M. \& Sayre, D. (2005). Proc. Natl Acad. Sci. USA, 102, 15343-15346.

Stangl, J., Mocuta, C., Chamard, V. \& Carbone, D. (2014). Nat. Mater. 8, 291-298. 
Thibault, P., Dierolf, M., Kewish, C. M., Menzel, A., Bunk, O. \& Pfeiffer, F. (2009). Phys. Rev. A, 80, 043813.

Thibault, P., Dierolf, M., Menzel, A., Bunk, O., David, C. \& Pfeiffer, F. (2008). Science, 321, 379-382.

Tomioka, K., Yoshimura, M. \& Fukui, T. (2012). Nature, 488, 189192.

Wallentin, J., Anttu, N., Asoli, D., Huffman, M., Åberg, I., Magnusson, M. H., Siefer, G., Fuss-Kailuweit, P., Dimroth, F., Witzigmann, B., Xu, H. Q., Samuelson, L., Deppert, K. \& Borgström, M. T. (2013). Science, 339, 1057-1060.
Wallentin, J. \& Borgström, M. T. (2011). J. Mater. Res. 26, 2142-2156. Wilke, R. N., Vassholz, M. \& Salditt, T. (2013). Acta Cryst. A69, 490497.

Wilke, R. N., Wallentin, J., Osterhoff, M., Pennicard, D., Zozulya, A., Sprung, M. \& Salditt, T. (2014). Acta Cryst. A70, 552-562.

Williams, G. J., Pfeifer, M. A., Vartanyants, I. A. \& Robinson, I. K. (2003). Phys. Rev. Lett. 90, 175501.

Yao, M., Huang, N., Cong, S., Chi, C.-Y., Seyedi, M. A., Lin, Y.-T., Cao, Y., Povinelli, M. L., Dapkus, P. D. \& Zhou, C. (2014). Nano Lett. 14, 3293-3303. 